\title{
Physical origin of pickup currents
}

\author{
V. M. Vasyliūnas \\ Max-Planck-Institut für Sonnensystemforschung, 37077 Göttingen, Germany \\ Correspondence to: V. M. Vasyliūnas (vasyliunas@mps.mpg.de)
}

Received: 4 November 2015 - Revised: 18 January 2016 - Accepted: 19 January 2016 - Published: 1 February 2016

\begin{abstract}
So-called pickup electric currents, associated with the ionization of neutral particles in the presence of a flowing plasma, are conventionally described as produced directly by differential displacement of ions and electrons as the result of acceleration and gyromotion under the action of the electric field $\boldsymbol{E}=-\boldsymbol{V} \times \boldsymbol{B} / c$. This is not the appropriate physical description for the case when the electron inertial length of the background plasma is small in comparison with the spatial scale of the system. The pickup process in this case does not directly produce a current, except for a small transient on the electron-gyroperiod timescale, which then decays exponentially at the ionization (momentum-loading) rate, as can be shown by an explicit solution of the equations. Rather, the plasma is first slowed down by the momentum loading; the spatially inhomogeneous velocity change then leads to a perturbation of the magnetic field, and the curl of the perturbed field is the current. The timescale for the development of the pickup current is not the ion gyroperiod (as the conventional description might suggest) but rather the Alfvén wave travel time over the spatial scale of the inhomogeneity.
\end{abstract}

Keywords. Magnetospheric physics (Magnetosphere interactions with satellites and rings)

\section{Introduction}

The ionization of neutral particles in the presence of flowing plasma has long been recognized as an important process in the interaction of planetary moons with their respective magnetospheres as well as of the solar wind with the interstellar medium (see, e.g., Saur et al., 2004; Thomas et al., 2004; Lee et al., 2009, and references therein). It is often called a pickup process because the newly created charged particles are subject to drift in the motional electric field $\boldsymbol{E}=-\boldsymbol{V} \times \boldsymbol{B} / \mathrm{c}$ and hence can be viewed as having been "picked up" by the perpendicular component of plasma bulk flow $\boldsymbol{V}$ (relative to neutral gas flow $\boldsymbol{V}_{\mathrm{n}}$ ). The $\boldsymbol{E} \times \boldsymbol{B} / c$ drift of the quasi-neutral plasma carries no net current, but it was pointed out by Goertz (1980) in a widely cited paper (and earlier by Cloutier, 1970) that, at each ionization, the mean position (guiding center) of the new ion/electron is displaced along $\pm \boldsymbol{E}$ by a distance equal to the respective gyroradius; summed over ongoing ionizations, this corresponds to a current density given by

$$
\boldsymbol{J}_{\perp} \simeq \dot{\rho} c^{2} \boldsymbol{E} / B^{2}
$$

or

$\boldsymbol{J} \times \boldsymbol{B} \simeq \dot{\rho} c\left(\boldsymbol{V}-\boldsymbol{V}_{\mathrm{n}}\right)$,

where $\dot{\rho}$ is the mass-weighted rate of ion-electron pair creation per unit volume and $\boldsymbol{E}$ is taken in the frame of reference of neutral gas flow. Equation (2) states that the Lorentz force of this current balances the momentum loading of plasma by ionization.

Following Goertz (1980), $\boldsymbol{J}$ given by Eq. (1) is called the pickup current and is widely regarded as a physical current created by the ionization process (e.g., Thomas et al., 2004, who state that it "flows in direct consequence of the initial displacement of each new ion and electron"). So described, the pickup current does not depend on presence or absence of any spatial variations, and its value is given by Eq. (1) without mention of the $\nabla \times \boldsymbol{B}$ required by Ampère's law. If time-varying ionization is considered, the description of the pickup process suggests that $\boldsymbol{J}$ and the magnetic field perturbation associated with it should evolve on the timescale of the order of an ion gyroperiod.

Although derived by a seemingly straightforward and convincing argument, this conventional view is not consistent with the long-established principle in MHD (Cowling, 1957; Dungey, 1958; Parker, 1996, 2000, 2007) that in plasmas, it is $\nabla \times \boldsymbol{B}$ that determines $\boldsymbol{J}$, not the other way around; more 
recently, this principle has been formulated on a firm mathematical basis, and its physical foundations have been clarified (Vasyliūnas, 2001, 2005a, b, 2011, 2012, and references therein). Here I consider how to resolve this inconsistency.

\section{Basic concepts and equations}

The essential property that distinguishes cosmic electrodynamics from the ordinary E\&M environment of the elementary laboratory is the large number (primarily because of the large spatial scales) of free charged particles and the consequent overriding importance of self-consistency between their distributions and the electromagnetic fields, the behavior of plasma being strongly constrained by charge quasineutrality. As shown in the references above, this applies to phenomena on spatial and temporal scales $\mathcal{L}$ and $\tau$ in the large-scale limit defined by

$\mathcal{L} \gg \lambda_{\mathrm{e}} \equiv c / \omega_{\mathrm{p}}$ and $\tau \gg 1 / \omega_{\mathrm{p}}$,

where $\omega_{\mathrm{p}} \equiv\left(4 \pi n_{\mathrm{e}} e^{2} / m_{\mathrm{e}}\right)^{1 / 2}$ is the plasma frequency and $\lambda_{\mathrm{e}} \equiv c / \omega_{\mathrm{p}}$ is the electron inertial length (numerical values: $\lambda_{\mathrm{e}}=5.3 \mathrm{~km} / \sqrt{n_{\mathrm{e}}}, \omega_{\mathrm{p}} / 2 \pi=\sqrt{n_{\mathrm{e}}} \times 9.0 \mathrm{kHz} ; n_{\mathrm{e}}$ in $\left.\mathrm{cm}^{-3}\right)$.

The principal consequences on these large scales are as follows:

1. $\boldsymbol{E}$ is determined by the generalized Ohm's law (in many cases, primarily by $\boldsymbol{V} \times \boldsymbol{B}$ or $\left.\boldsymbol{V}_{\mathrm{e}} \times \boldsymbol{B}\right)$;

2. $\boldsymbol{J}$ is determined by $\nabla \times \boldsymbol{B}$;

3. the time evolution of $\boldsymbol{B}$ is determined by $\nabla \times \boldsymbol{E}$;

4. in a quasi-steady state (which in general can exist only if the system is sufficiently stable), $\boldsymbol{B}$ is determined by the requirement that the magnetic stress $(\nabla \times \boldsymbol{B}) \times \boldsymbol{B} / 4 \pi$ balance the mechanical stress.

To understand physical origins and causal relations, however, the exact equations must be taken as the starting point and the large-scale approximation invoked afterwards. In particular, it is necessary to include the displacement-current term in Maxwell's equations, which plays a key role in the relation between current and magnetic field (Vasyliūnas, 2005b; Song and Lysak, 2006).

\subsection{Simplified key equations}

To describe how the ionization process gives rise to a current, I consider time-dependent evolutionary equations for $\boldsymbol{J}$ and related quantities, neglecting spatial gradients (these play no role in the conventional view, in which pickup currents can be produced even in a quasi-uniform configuration). In the following, "+..." stands for spatial-gradient terms not shown explicitly, $\dot{\rho}$ is the mass-weighted total ionization rate (including photoionization, electron-impact ionization, and charge exchange), and $\dot{\rho}_{x}=m_{i} \dot{n}_{x}$ is the rate for charge exchange only. It is assumed that $\boldsymbol{B}=\boldsymbol{B}_{0}+\delta \boldsymbol{B}$ and $|\delta \boldsymbol{B}| \ll\left|\boldsymbol{B}_{0}\right|$, with $\boldsymbol{B}_{0}$ a (nearly) constant background field.

$\partial \boldsymbol{E} / \partial t=-4 \pi \boldsymbol{J}+c \nabla \times \boldsymbol{B}$

(difference between $\boldsymbol{J}$ and $(c / 4 \pi) \nabla \times \boldsymbol{B}$ changes $\boldsymbol{E})$,

$$
\begin{aligned}
\partial \boldsymbol{J} / \partial t= & \left(\omega_{\mathrm{p}}^{2} / 4 \pi\right)(\boldsymbol{E}+\boldsymbol{V} \times \boldsymbol{B} / c)-\boldsymbol{J} \times\left(\boldsymbol{\Omega}_{\mathrm{e}}-\boldsymbol{\Omega}_{i}\right) \\
& +\ldots-\dot{n}_{x} e\left(\boldsymbol{V}-\boldsymbol{V}_{\mathrm{n}}\right)
\end{aligned}
$$

(deviation of $\boldsymbol{E}$ from the value given by generalized Ohm's law changes $\boldsymbol{J}$, on timescale $\sim \omega_{\mathrm{p}}^{-1}$ ),

$\partial \boldsymbol{B} / \partial t=-c \nabla \times \boldsymbol{E}$

( $\boldsymbol{B}$ changed only by spatial variation $\nabla \times \boldsymbol{E} \neq 0$ ),

$\rho \partial \boldsymbol{V} / \partial t+\ldots=\boldsymbol{J} \times \boldsymbol{B} / c-\dot{\rho}\left(\boldsymbol{V}-\boldsymbol{V}_{\mathrm{n}}\right)$

(bulk flow changed only by stress imbalance).

The last terms in Eqs. (5) and (7) represent changes in $\boldsymbol{J}$ and $\boldsymbol{V}$, respectively, due to ionization processes; $\boldsymbol{V}$ is affected by ionization of all types, but $\boldsymbol{J}$ is affected, to first approximation, only by charge exchange (which changes the velocity of an ion but not an electron, whereas photoionization or electron-impact ionization produces an ion-electron pair, both moving at nearly the same velocity).

\subsection{Solution for average current}

Differentiating Eq. (5) and using Eqs. (4) and (7) to eliminate time derivatives of $\boldsymbol{E}$ and $\boldsymbol{V}$ gives

$$
\begin{aligned}
& \partial^{2} \boldsymbol{J} / \partial t^{2}+\left(\omega_{\mathrm{p}}^{2}+\Omega_{\mathrm{e}} \Omega_{i}\right) \boldsymbol{J} \\
& +(\dot{\rho} / \rho) \Omega_{i} \boldsymbol{J} \times \hat{\boldsymbol{B}}+(\partial \boldsymbol{J} / \partial t) \times\left(\boldsymbol{\Omega}_{\mathrm{e}}-\boldsymbol{\Omega}_{\boldsymbol{i}}\right) \\
& +\ldots \simeq\left[(\dot{\rho} / \rho) \Omega_{\mathrm{e}} \hat{\boldsymbol{B}} \times+\left(\dot{\rho} \dot{\rho}_{x} / \rho^{2}\right)\right] n e\left(\boldsymbol{V}-\boldsymbol{V}_{\mathrm{n}}\right),
\end{aligned}
$$

which is a harmonic-oscillator equation for $\boldsymbol{J}$, with an oscillation frequency of $\sim \omega_{\mathrm{p}}$ ( \pm gyrofrequency corrections, e.g., Vasyliūnas, 2001) and source terms proportional to $\dot{\rho}$. Averaging Eq. (8) over the oscillations to lowest order in $\dot{\rho} / \rho$ gives $\boldsymbol{J}$ for timescales that are long compared to $\omega_{\mathrm{p}}{ }^{-1}$ but short compared to long-term evolution:

$$
\begin{aligned}
\left\langle\boldsymbol{J}_{\perp}\right\rangle & \simeq(\dot{\rho} / \rho) \Omega_{\mathrm{e}} \hat{\boldsymbol{B}} \times n e\left(\boldsymbol{V}-\boldsymbol{V}_{\mathrm{n}}\right) /\left(\omega_{\mathrm{p}}^{2}+\Omega_{\mathrm{e}} \Omega_{i}\right) \\
& \simeq\left(\dot{\rho} c^{2} \boldsymbol{E} / B^{2}\right)\left[V_{\mathrm{A}}^{2} /\left(c^{2}+V_{\mathrm{A}}^{2}\right)\right]
\end{aligned}
$$

or equivalently

$$
\langle\boldsymbol{J}\rangle \times \boldsymbol{B} \simeq \dot{\rho} c\left(\boldsymbol{V}-\boldsymbol{V}_{\mathrm{n}}\right)\left[V_{\mathrm{A}}^{2} /\left(c^{2}+V_{\mathrm{A}}^{2}\right)\right],
$$

values smaller than those in Eqs. (1) and (2) by the factor $\sim V_{\mathrm{A}}^{2} / c^{2} \ll 1$. The actual current that results from ionization in a quasi-uniform medium is thus much smaller than 
the conventional pickup current, and its Lorentz force does not balance momentum loading: with Eq. (10) inserted, the momentum Eq. (7) becomes

$$
\begin{aligned}
\rho \partial \boldsymbol{V} / \partial t & \simeq-\dot{\rho}\left(\boldsymbol{V}-\boldsymbol{V}_{\mathrm{n}}\right)\left[c^{2} /\left(c^{2}+V_{\mathrm{A}}^{2}\right)\right] \\
& \simeq-\dot{\rho}\left(\boldsymbol{V}-\boldsymbol{V}_{\mathrm{n}}\right),
\end{aligned}
$$

showing that $\boldsymbol{V}-\boldsymbol{V}_{\mathrm{n}}$ and therefore also, from Eq. (9), $\left.\langle\boldsymbol{J}\rangle\right)$ both decay at the rate $\dot{\rho} / \rho$.

The equations of the ionospheric neutral-wind dynamo problem, which describe the dynamics of ionospheric plasma interacting with neutrals by collisions, are identical with Eqs. (4)-(7) for the pickup-ion problem (dynamics of plasma interacting with neutrals by ionization) if one replaces collision frequencies with ionization rates

$$
\begin{array}{lll}
\nu_{\text {in }} & \rightarrow \dot{\rho} / \rho & \text { (momentum loading) } \\
\nu_{\text {en }} \rightarrow \dot{\rho} / \rho-\dot{\rho}_{x} / \rho & \text { (mass loading) }
\end{array}
$$

(Vasyliūnas, 2012). ("Mass loading" is commonly used to denote all ionization processes other than charge exchange which add net mass to the plasma; since all ionization processes affect plasma momentum as long as $\boldsymbol{V}_{\mathrm{n}} \neq \boldsymbol{V}$, mass loading is actually a subset of "momentum loading.") The mathematical problem is thus the same for pickup currents and for currents created by neutral winds. For the latter, Vasyliūnas (2012) obtained a solution of the initial-value problem for the counterpart of Eqs. (4)-(7) and showed that a small transient $\boldsymbol{J}$ appears on a timescale $O\left(\Omega_{\mathrm{e}}^{-1}\right)$ but then decays with time as described by Eq. (11).

\subsection{Root of the inconsistency}

What does the conventional derivation of pickup currents overlook? There is an additional current from polarization drifts, given by

$\rho \partial \boldsymbol{V} / \partial t \equiv \boldsymbol{J}_{\mathrm{pol}} \times \boldsymbol{B} / c$,

which can also be written as

$\boldsymbol{J}_{\mathrm{pol}} \simeq\left(c^{2} / 4 \pi V_{\mathrm{A}}^{2}\right) \partial \boldsymbol{E} / \partial t$

(mentioned but not further discussed by Goertz, 1980). Inserting Eq. (13) into the momentum Eq. (7) gives

$\dot{\rho}\left(\boldsymbol{V}-\boldsymbol{V}_{\mathrm{n}}\right)=\left(\boldsymbol{J}-\boldsymbol{J}_{\mathrm{pol}}\right) \times \boldsymbol{B} / c$,

and inserting $\partial \boldsymbol{E} / \partial t$ from Eq. (14) into Maxwell's Eq. (4) gives

$\nabla \times \boldsymbol{B}=(4 \pi / c)\left[\boldsymbol{J}+\left(V_{\mathrm{A}}^{2} / c^{2}\right) \boldsymbol{J}_{\mathrm{pol}}\right]$.

Equation (10) follows from Eqs. (15) and (16) when $\nabla \times \boldsymbol{B}$ is negligible.

\section{Physical description}

The argument of Sect. 2.3 can be restated in simple physical terms: as long as no sufficient $\nabla \times \boldsymbol{B}$ has developed, the differential displacement of newly created ions and electrons to produce the pickup current also acts to change $\boldsymbol{E}$. The resulting $\partial \boldsymbol{E} / \partial t$ implies polarization drifts of all the plasma particles, not just the few newly ionized ones. The polarization current is thus large enough to (almost) cancel the pickup current, so that the net current is that given by Eqs. (9)-(10) instead of Eqs. (1)-(2).

In more fundamental terms, if a quasi-uniform configuration is assumed and spatial gradients neglected, there is no external force that can balance momentum loading. The effect of ionization and consequent momentum loading on an initially imposed flow of plasma relative to neutrals is then simply to reduce the difference $\boldsymbol{V}-\boldsymbol{V}_{\mathrm{n}}$, until plasma and neutrals flow together and electric fields as well as currents disappear. If this does not happen (and, to my knowledge, it has never yet been observed to happen in a magnetospheric or heliospheric context), there must be forces tending to maintain the flow difference between plasma and neutrals.

How a sustained current may develop from the pickup process can be illustrated by an order-of-magnitude argument (see Vasyliūnas, 2005b, 2011, for more detailed discussion): initially, with $|\boldsymbol{J} \times \boldsymbol{B}| \ll \dot{\rho} c V$, momentum loading from pickup slows down the plasma flow:

$\delta V \sim-(\dot{\rho} / \rho) V t$.

Reduced $\boldsymbol{V}$ implies reduced $\boldsymbol{E}$. If slowdown varies on the spatial scale $\mathcal{L}$, outside which there is no slowdown and no reduced $\boldsymbol{E}$ (because the flow is maintained by other forces), then $\nabla \times \boldsymbol{E} \neq 0$ and $\boldsymbol{B}$ changes:

$\delta B \sim(B \delta V / \mathcal{L}) t \sim(\dot{\rho} / \rho) t^{2}(V B / \mathcal{L})$.

The resulting Lorentz force $(\nabla \times \boldsymbol{B}) \times \boldsymbol{B} / 4 \pi$ increases with time and balances momentum loading after a time $t$ given by

$\dot{\rho} V \sim B \delta B / 4 \pi \mathcal{L} \sim(\dot{\rho} / \rho) t^{2}\left(V B^{2} / 4 \pi \mathcal{L}^{2}\right)$

or

$t^{2} \sim\left(4 \pi \rho / B^{2}\right) \mathcal{L}^{2}=\mathcal{L}^{2} / V_{\mathrm{A}}^{2}$.

Thus, after a time on the order of Alfvén wave travel time, $\partial \boldsymbol{V} / \partial t \rightarrow 0$, the Lorentz force balances momentum loading, and $\boldsymbol{J}$ now has the conventional value given by Eqs. (1)-(2). (In most magnetospheric applications, the Alfvén wave travel time is much shorter than the momentum-loading timescale $\rho / \dot{\rho}$.) 


\section{Conclusions}

1. Pickup current as a physically distinct kind of electric current does not exist. The pickup process does not by itself produce an electric current, except for a small transient $O\left(V_{\mathrm{A}}^{2} / c^{2}\right)$ that decays at the rate $\dot{\rho} / \rho$.

2. The primary direct effect of the pickup process is to slow down the plasma flow (relative to the neutrals). A spatial inhomogeneity of the slowdown (e.g., momentum loading confined to the equatorial region) together with forces tending to maintain the plasma flow elsewhere (e.g., corotation imposed at the ionosphere) produces a deformation of the magnetic field, and the curl of the deformed field gives the current.

3. The timescale for the development of this current is the Alfvén wave travel time over the spatial scale of the inhomogeneity.

4. The $\boldsymbol{J} \times \boldsymbol{B} / \boldsymbol{c}$ force opposes the slowdown. If and when a steady state is reached, $\boldsymbol{J} \times \boldsymbol{B} / c$ must balance the sum of all the mechanical stresses acting on the plasma. The so-called pickup current corresponds merely to the part of $\boldsymbol{J} \times \boldsymbol{B} / c$ that balances the momentum loading.

Acknowledgements. I am grateful to J. R. Jokipii and J. Saur for useful discussions and to the referee for suggestions that significantly improved the presentation.

The article processing charges for this open-access publication were covered by the Max Planck Society.

The topical editor, Lars Blomberg, thanks one anonymous referee for help in evaluating this paper.

\section{References}

Cloutier, P. A.: Dynamics of the interaction of the solar wind with a planetary atmosphere, Radio Sci., 5, 387-389, 1970.

Cowling, T. G.: Magnetohydrodynamics, Interscience Publishers, Inc., New York, Section 1.3, 1957.
Dungey, J. W.: Cosmic Electrodynamics, Cambridge University Press, London, p. 10, 1958.

Goertz, C. K.: Io's interaction with the plasma torus, J. Geophys. Res., 85, 2949-2956, 1980.

Lee, M. A., Fahr, H. J., Kucharek, H., Moebius, E., Prested, C., Schwadron, N. A., and Wu, P.: Physical processes in the outer heliosphere, Space Sci. Rev., 146, 275-294, doi:10.1007/s11214009-9522-9, 2009.

Parker, E. N.: The alternative paradigm for magnetospheric physics, J. Geophys. Res., 101, 10587-10625, 1996.

Parker, E. N.: Newton, Maxwell, and magnetospheric physics, in: Magnetospheric Current Systems, edited by: Ohtani, S.-I., Fujii, R., Hesse, M., and Lysak, R. L., Geophysical Monograph 118, American Geophysical Union, Washington, D.C., 1-10, 2000.

Parker, E. N.: Conversations on Electric and Magnetic Fields in the Cosmos, Princeton University Press, Princeton, New Jersey, 2007.

Saur, J., Neubauer, F. M., Connerney, J. E. P., Zarka, P., and Kivelson, M. G.: Plasma interaction of Io with its plasma torus, in: Jupiter: The Planet, Satellites and Magnetosphere, edited by: Bagenal, F., Dowling, T. E., and McKinnon, W. B., Cambridge University Press, Cambridge, UK, 537-560, 2004.

Song, Y. and Lysak, R. L.: Displacement current and the generation of parallel electric fields, Phys. Rev. Lett., 96, 145002, doi:10.1103/PhysRevLett.96.145002, 2006.

Thomas, N., Bagenal, F., Hill, T. W, and Wilson, J. K.: The Io neutral clouds and plasma torus, in: Jupiter: The Planet, Satellites and Magnetosphere, edited by Bagenal, F., Dowling, T. E., and McKinnon, W. B., Cambridge University Press, Cambridge, UK, 561-591, 2004.

Vasyliūnas, V. M.: Electric field and plasma flow: What drives what?, Geophys. Res. Lett., 28, 2177-2180, 2001.

Vasyliūnas, V. M.: Time evolution of electric fields and currents and the generalized Ohm's law, Ann. Geophys., 23, 1347-1354, doi:10.5194/angeo-23-1347-2005, 2005a.

Vasyliūnas, V. M.: Relation between magnetic fields and electric currents in plasmas, Ann. Geophys., 23, 2589-2597, doi:10.5194/angeo-23-2589-2005, 2005b.

Vasyliūnas, V. M.: Physics of magnetospheric variability, Space Sci. Rev., 158, 91-118, doi:10.1007/s11214-010-9696-1, 2011.

Vasyliūnas, V. M.: The physical basis of ionospheric electrodynamics, Ann. Geophys., 30, 357-369, doi:10.5194/angeo-30-3572012, 2012. 